\title{
Quantitative Analysis of Fetal Actocardiogram: Update
}

\section{Kazuo Maeda*}

Department of Obstetrics and Gynecology (Emeritus), Tottori University Medical School, Yonago, Japan

\begin{abstract}
Aims: The actocardiogram (ACG), which recorded fetal heart rate (FHR) and movements, was quantitatively analyzed, due to its advantage to the FHR patterns diagnosis, to recognize the effect of fetal movement on FHR, to solve controversial FHR problems, to correctly evaluate fetal disorders, and to evaluate the loss of variability and acceleration.

Methods: FHR changes were diagnosed by the FHR score, fetal movements were evaluated by 4 ACG parameters, fetal behavior and abnormal FHR were quantitatively determined, physiologic sinusoidal FHR was diagnosed by the ACG and FHR frequency analysis, the developing mechanism of FHR acceleration and variability were studied to diagnose the brain damage in the loss of FHR variability. 1.3 Results: The FHR score, neural network analysis and A/B ratio predicted short and long term outcomes in the 1st stage of labor or even in pregnancy. The ACG and frequency analysis differentiated physiologic sinusoidal from the true one, Contoversial problems in FHR were solved by the quantitative ACG analysis. The loss of FHR variability was the sign of fetal brain damage even in fetal non-hypoxic insults.
\end{abstract}

Results: The FHR score, neural network analysis and A/B ratio predicted short and long term outcomes in the 1st stage of labor or even in pregnancy. The ACG and frequency analysis differentiated physiologic sinusoidal from the true one, Contoversial problems in FHR were solved by the quantitative ACG analysis. The loss of FHR variability was the sign of fetal brain damage even in fetal non-hypoxic insults.

Conclusion: Quantitative analyses of FHR and fetal movements in ACG were indispensable in the fetal diagnosis even in general insults. Since cerebral palsy (CP) could develop in the loss of FHR variability, C-section is recommended to perform before the loss of FHR variability.

Keywords: FHR; Fetal movement; Actocardiogram; FHR score; A/B ratio; Outcome prediction; FHR variability; Acceleration; Fetal disorders

\section{Definition}

Actocardiogram (ACG): The ACG simultaneously traced FHR curve and fetal movement signals. The $\mathrm{MHz}$ level continuous wave (CW) ultrasound was used, of which SPTA intensity was as weak as $1 \mathrm{~mW} / \mathrm{cm}^{2}$. The fetal movement Doppler signals obtained by $2 \mathrm{MHz}$ source ultrasound was $20-50 \mathrm{~Hz}$. The ultrasound detected Doppler fetal heart beat signals at the same time, of which Doppler frequency was 100 or more HZ, i.e. single ultrasonic probe detected two phenomena, FHR and fetal movements. Fetal movement Doppler signal was separated by a band-pass filter, and formed low frequency spikes, of which amplitude was parallel to that of fetal movement (Figures 1 and 2) [1]. Uterine contraction was also simultaneously recorded to prepare the CTG function.

Cardiotocogram (CTG): is simultaneous record of FHR and uterine contraction curves.

Fetal movements: The ACG motion signal developed by fetal trunk movements, because the ultrasound targeted fetal heart. The motion was fetal respiratory movements (continuous and periodic respiratory changes), fetal hiccupping, and the trunk motion caused by conducted extremity movements, and mouthing movements [2].

Fetal heart rate (FHR): More than $100 \mathrm{~Hz}$ Doppler signals developed by fetal heart beats were introduced into an autocorrelation FHR meter, which detected the FHR up to 210 beats per minutes (bpm) [1].

Uterine contraction: It is simultaneously recorded on the ACG chart usually by external tocodynamometry, by which no intrauterine pressure is evaluated, but used for the diagnosis of late FHR deceleration.
FHR baseline: Continuous FHR tracing without acceleration or deceleration. Normal baseline is 110 to $159 \mathrm{bpm}$.

Fetal bradycardia: The baseline FHR less than $110 \mathrm{bpm}$.

Fetal tachycardia: The baseline FHR of $160 \mathrm{bpm}$ or more.

FHR variability: Minor variation of FHR baseline (long term variability, LTV) in the FHR recorded by ultrasonic Doppler autocorrelation FHR meter. The LTV amplitude is normal when it is 5 to $24 \mathrm{bpm}$.

Acceleration: Transient FHR increase for 10 or more bpm and 10 or longer sec duration is normal before 30 weeks of pregnancy, and 15 $\mathrm{bpm}$ and $15 \mathrm{sec}$ or more is normal in 30 or more weeks of pregnancy.

Deceleration: Fifteen or more bpm deep and 15 or more sec long transient FHR decrease. V-shaped slow deceleration is a periodic deceleration including early (ED) and late (LD) decelerations comparing to uterine contraction. U-shaped irregular deceleration with sudden decrease and recovery is variable deceleration, divided into mild, moderate and severe variable deceleration (MVD and SVD). The LD and SVD are pathological in the pattern classification.

${ }^{*}$ Corresponding author: Kazuo Maeda, Department of Obstetrics and Gynecology (Emeritus), Tottori University Medical School, Nadamachi, Yonago, Tottoriken, 6830835, Japan, Tel: 81-859-22-6856; Fax: 81-859-22-6856; E-mail: maeda@mocha.ocn.ne.jp

Received December 04, 2013; Accepted December 18, 2013; Published December 25, 2013

Citation: Maeda K (2013) Quantitative Analysis of Fetal Actocardiogram: Update. $\mathrm{J}$ Health Med Informat 4: 140. doi:10.4172/2157-7420.1000140

Copyright: (C) 2013 Maeda K. This is an open-access article distributed under the terms of the Creative Commons Attribution License, which permits unrestricted use, distribution, and reproduction in any medium, provided the original author and source are credited. 
A/B ratio: Total sum of acceleration duration ratio to the total sum of fetal movement duration in a study period.

\section{Introduction}

Although fetal movements were evaluated by maternal perception or abdominal motion detected by mechanical actograph at abdominal surface in old time, the results were insufficient to scientific fetal movement study [3]. A direct detection of fetal movement at fetal surface was required, and the device was invented by Maeda in 1984 [1]. Although the CTG was diagnosed mainly by FHR pattern classification [4], several vague problems and diagnostic difficulty were found in the past. It was, therefore, necessary to analyze the CTG with quantified techniques and numeric evaluation, and the result was the creation of FHR score [3-6]. Still there was the difficulty of differentiation of resting fetal state from non-reactive FHR, the differentiation of physiological sinusoidal FHR from true ominous sinusoidal FHR, and so on, which were related to the lack of fetal movement study. Although some problems were solve by the real-time B-mode ultrasound [7], most diagnostic difficulties were solved by the handling of ultrasonic Doppler fetal movement signals [1]. The prototype actocardiograph was handmade by Maeda (Figures 1 and 2) and reported after the confirmation of its basic properties [1].

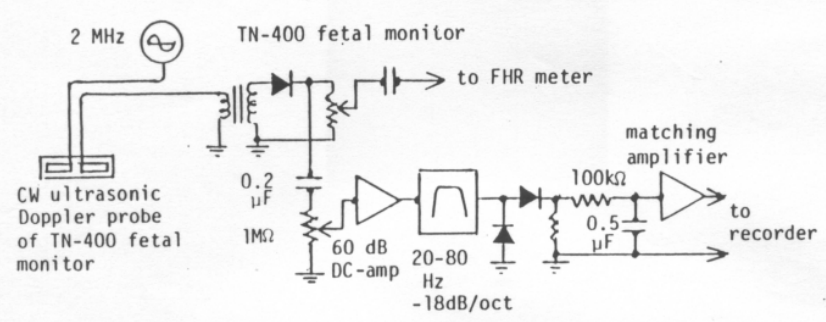

Figure 1: The circuit to record fetal movement in the first actocardiogam, which was hand-made by Maeda in 1983 [1].

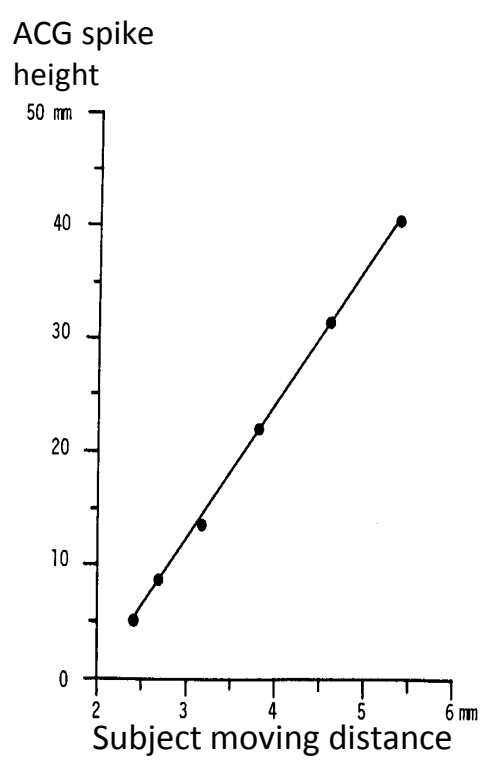

Figure 2: The amplitude of fetal movement spikes recorded at fetal ACG chart was linearly parallel to the moving distance of ACG subject (a steel ball moved in the water). The ACG precisely records the movement of the subject [1].

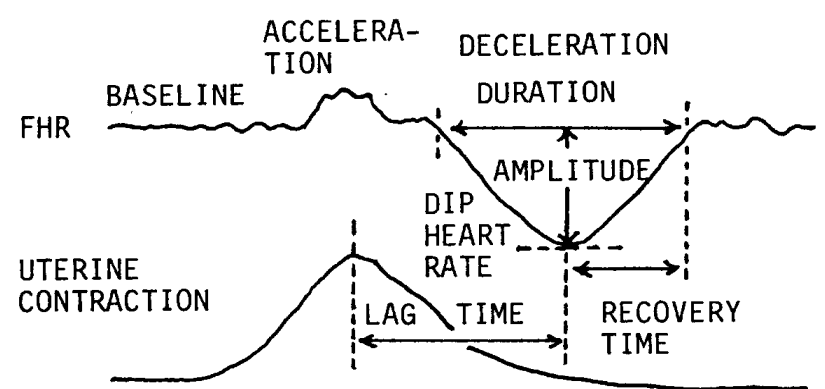

Figure 3: Quantified analysis of fetal heart rate change to determine the FHR score in non-interventional labors in 1969 [3], where FHR was manually analyzed, and it was computerized in 1970 s.

\begin{tabular}{|c|c|c|}
\hline FHR & Apgar score $<7(\%)$ & Evaluation score \\
\hline \multirow{2}{*}{$\begin{array}{l}\text { Baseline FHR: } 110- \\
\text { 130/160-180 bpm }\end{array}$} & $11 / 40(28)$ & 1 \\
\hline & $>180$ or $<110$ bpm (70) & 3 \\
\hline \multirow{7}{*}{$\begin{array}{l}\text { Deceleration dip } \\
\text { hear rate }\end{array}$} & $<100$ bpm 13/35 (37) & 2 \\
\hline & amplitude>50 bpm 11/22 (50) & 2 \\
\hline & lag time $>40 \sec 10 / 14(71)$ & 2 \\
\hline & recovery time $>40 \sec 12 / 19(63)$ & 3 \\
\hline & duration >60 sec 15/21(71) & 3 \\
\hline & no acceleration 21/47 (45) & 2 \\
\hline & W shape, loss variability $11 / 11(100)$ & 4 \\
\hline
\end{tabular}

Table 1: FHR score is the sum of evaluation scores in $5 \mathrm{~min}$, which were determined by the incidence of low Apgar cases [3].

\section{Methods}

The FHR was quantified by the FHR score, and fetal movements by the ACG. There were various commercial ACG models after the prototype ACG (Figure 1), including MT-320, MT-325, MT-332, MT-333U, MT-430, MT-516, MT-517, MT-522 and MT-540 (TOITU, Tokyo). Recent ACG works were mainly studied by the ACG records offered by friends of the author, therefore, deep appreciation is expressed to the kind offering. Four parameters were quantitatively analyzed in the quantified studies on fetal behavior [2]. Physiologic sinusoidal FHR was differentiated from true ominous one by fetal periodic respiratory and swallowing movements [8]. Developmental mechanism of acceleration and LTV was studied. Short and long fetal outcomes were studied by the $A / B$ ratio [9]. New prognostic value of the loss of LTV was clarified in this report.

\section{Results}

\section{Quantitative diagnosis of CTG by FHR score}

It was the first trial in 1960s to quantitatively analyze intrapartum CTG instead of visual pattern classification (Figure 3). The percentage of low Apgar score gave evaluation scores from 1 to 4 in abnormal FHR changes (Table 1). The method was statistically a goodness measure. The evaluation scores were summarized in $5 \mathrm{~min}$ to obtain the FHR score [2], which was compared to Apgar scores and significant correlation was found (Figure 4) [5]. The CTG analysis with FHR score were programmed in the computer for automated CTG diagnosis $[5,6,10]$.

Fetal state is abnormal, if FHR score is 10-19, and highly abnormal if the score is 20 or more. Thus the FHR score comprehensively evaluates fetal status. 
10

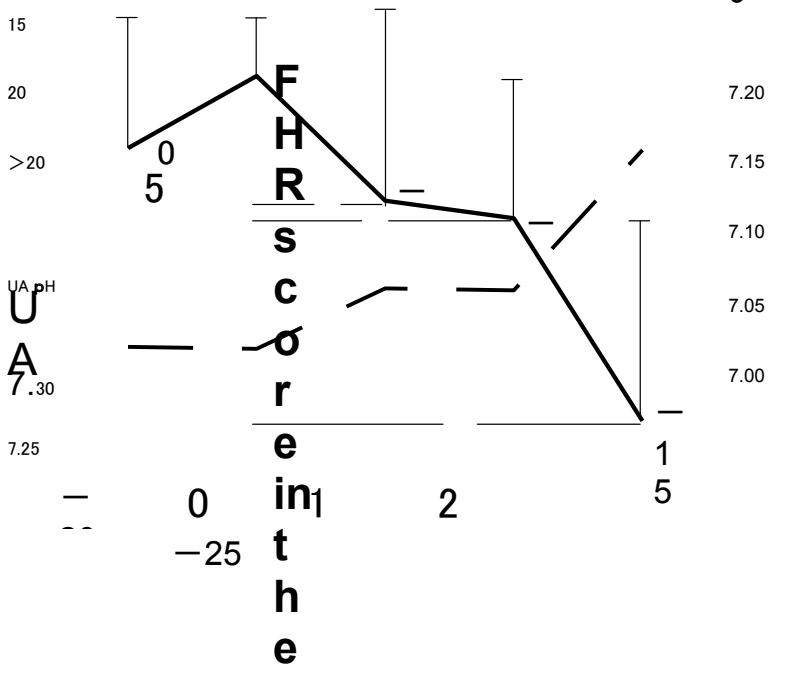

Figure 4: (Upper figure) Highest FHR score in the 1st stage of labor (X) was closely correlated to the Apgar score $(Y), Y=9.361-0.335 X, R 2=0.84, p<0.05$. (Lower figure) Also the umbilical arterial blood $\mathrm{pH}(\mathrm{Y})$ was correlated with $\mathrm{FHR}$ score $(X), Y=7.31-0.01 X, R=0.85, p=0.024$. Fetal outcome is predicted by the FHR score even in early stage of labor [9].

\section{Other quantitative computerized CTG analyses}

Artificial neural network computer analysis: Objective decision was made by neural network analysis, because of the network training with actual FHR data of known outcome cases instead of experts' knowledge [10].

Training of neural network computer: The neural network softwear was trained by 8 FHR data (baseline FHR, variability, sinusoidal heat rate, dip number, dip duration, nadir FHR, recovery time and lag time) of 3 normal, 3 intermediate and 14 pathological outcome cases for 10,000 times by Noguchi and colleagues to obtain $100 \%$ correct internal check.

Diagnosis of neural network: Trained softwear was installed into other computers to diagnose new cases with three outcome probabilities to be normal, suspicios and pathological.

Accuracy of neural network diagnosis: It was confirmed by the FHR score, which was high in pathologic, modrate in suspicios and low in normal outcome probabilities.

Frequency analysis of FHR traces: Since FHR record is composed of various frequency signs, e.g. straight baseline, the acceleration, deceleation, sinusoidal change and long term variability,and so on, the fast Furier transform (FFT) frequenxcy analysis was suitable in the quantitative FHR analysis.

Main subject of the FFT frequency analysis was the differentiaion of true ominus sinusoidal FHR from physiological benign sinusoidal change. The true ominous sinusoidal FHR was diagnosed when the low frequency area to total spectral area $(\mathrm{La} / \mathrm{Ta})$ ratio was $39 \%$ or more and at the same time peak power spetral densiy (PPSD) was 300 or more $\mathrm{bpm}^{2} / \mathrm{Hz}$, while the values were lower in the physiological false sinusoidal FHR than true one [11]. In addition, the loss of LTV less than resting fetal state was diagnosed when the La/Ta was less than $15 \%$ and the PPSD was less than $60 \mathrm{bpm}^{2} / \mathrm{Hz}$ [12].

\section{Quantified diagnoasis of fetal behavior by the ACG}

Visual diagnosis of fetal behavior with actocardiogram: Resting fetal state is visually diagnosed when there is no acceleation, but no fetal movement burst was registered, and baseline variability is preserved. Active fetal state is characterized by fequent FHR aceleation, which are synchronized to fetal movement bursts, and baseline variability is as large as 5 to $24 \mathrm{bpm}$. Highly active fetal state is diagnsed by long duraion of acelertion synchronized to long lasting fetal movements. The longest acceleration lasted for about 5 minutes. Intermediate state showed rare FHR accelerations but not zero and accompanied rare fetal movement burst [2].

Quantified analysis of fetal behavior: The fetal ACG was analyzed by four parameters as follows;

Four fetal movement parameters

1. Burst duration (sec): Mean duration of fetal movement burst

2. Occupancy (\%): Percentage of the sum of movement burst durations against the whole analysis time.

3. Frequency (cycles per minute, cpm): The incidence of movement bursts in one min

4. A/B ratio (\%): The ratio of the sum of acceleeration durations to the sum of movement burst durations.

Four parameters' values were determined in the fetal ACGs of nomal fetuses in the late stage of pregnancy (Table 2). Fetal behavior will be determined by 4 parameters in new cases. The importance of $\mathrm{A} / \mathrm{B}$ ratio was confirmed, because it is not influenced by fetal behavior in the evaluation of fetal statuses.

Difference of ACG parameters in normal fetus and fetal hypoxia: There was significant difference of quantified parameters between normal and hypoxic fetuses, i.e. occupancy was $32.67 \%$ in normal fetus and $10.00 \%$ in hypoxia, frequency was 0.65 and $0.24 \mathrm{cpm}, \mathrm{A} / \mathrm{B}$ ratio was 1.03 and 0 , respectively [13]

Differentiation of physiologic sinusoidal FHR from true omioua one by the ACG

The CTG hardly differentiated physiologic sinusoidal FHR fom the truly ominous one. A physiologic one was easily diagnosed to be harmless in fetal ACG when the periodic fetal movements synchonized to the sine wave-like FHR, e.g. periodic fetal respiratory or mouth movements provocate physiologic sinusoidal FHR (Figure 5) [8].

\section{Developmental mechanism of FHR acceleration and LTV studied by the ACG}

Acceleration: A motion increases heart rate in human physiology. It was clear also in the fetus, i.e. a fetal movement burst accompanies triangular FHR acceleration (Figure 6), where the movement preceds acceleration for approx. $7 \mathrm{sec}$. The acceleration was lost in the non-

\begin{tabular}{|l|l|l|l|l|l|}
\hline $\begin{array}{l}\text { Behavioral } \\
\text { States }\end{array}$ & $\begin{array}{l}\text { Duration } \\
\text { (sec) }\end{array}$ & Occupancy & $\begin{array}{l}\text { (\%I Fequency } \\
\text { (cpm) }\end{array}$ & $\begin{array}{l}\text { A/B ratio } \\
(\mathbf{\%})\end{array}$ & $\mathbf{N}$ \\
\hline Resting & 0 & 0 & 0 & $0 / 0$ & 12 \\
\hline Active & $29.7 \pm 10.3$ & $32.7 \pm 14.8$ & $0.7 \pm 0.2$ & $1.4 \pm 0.4$ & 14 \\
\hline Intermediate & $17.7 \pm 3.7$ & $6.4 \pm 1.8$ & $0.2 \pm 0.03$ & $1.2 \pm 0.3$ & 5 \\
\hline Highly active & $88.6 \pm 14.6$ & $44.1 \pm 5.3$ & $0.5 \pm 0.3$ & $1.2 \pm 0.1$ & 3 \\
\hline
\end{tabular}

Table 2: Quantitative values in four fetal behavioral states detected by fetal ACGs The behavioral state of a fetus will be determined by comparing the ACG data to this table. The $A / B$ value was constant among three behavioral states of normal fetus, i.e. the $A / B$ ratio will be feasible to evaluate the fetal well-being. 


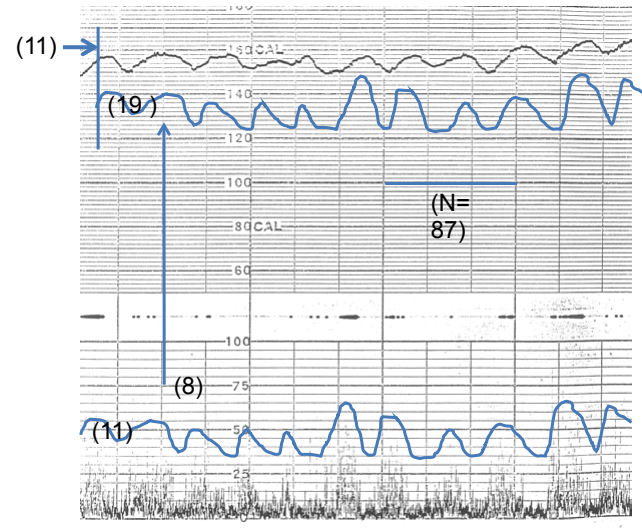

Figure 5: Physiologic benign sinusoidal FHR synchonized with the envelope of fetal periodic respiaory movements, when the movement envelope was delayed for approx. $7 \mathrm{sec}$, because the correlation of FHR and fetal movement was largest when the movement was delayed for $7 \mathrm{sec}$. Truly ominous sinusoidal FHR accompnied no periodic fetal movement.

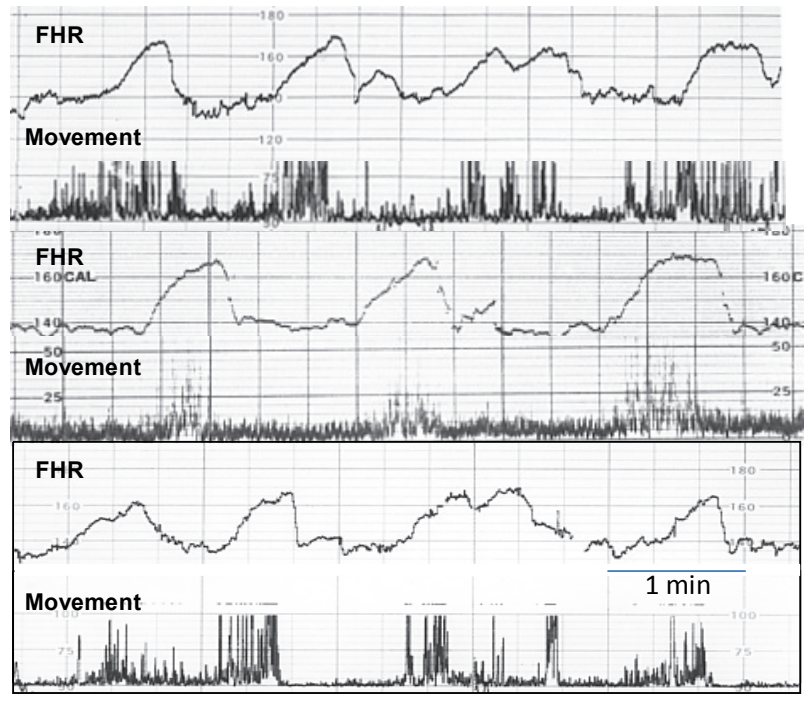

Figure 6: Three cases of triangular FHR accelerations synchronized to the feta movement bursts (grouped fetal movements).

reactive FHR, whicn follows severely hypoxic FHR changes some days later, including the braycardia, late deceleration and the loss of variability [14].

\section{The mechanism to produce triangular shape of acceleration}

A triangular curve developed in electric wave bursts after passing thriugh an integral circuit with 7 sec delay time constant. Also triangular heart rate curves developed in the 1 min lasting continuos leg motions in an adult [15]. The electric and physiologic simulations explained the triangular FHR developing mechanism, which was the brain excitation in the mid brain by the movements, i.e., Terao et al. [16] reported that the acceleration developed in the mid brain.

\section{The mechanism to develop FHR variability in the brain}

A repeated moderate fetal movements provoked synchronized FHR accelerations (Figure 6), and minor fetal movements also provoked FHR baseine changes (Figure 7). These facts indicate that the long term variability (LTV) develops as the response of fetal brain to minor fetal movements. Since the FHR delay was $7 \mathrm{sec}$, the location to respond the movement is the same as FHR acceleration, i.e. it is the mid brain [16].

\section{Prenatal quantitative diagnosis of outcome with the $A / B$} ratios of ACG

The $\mathrm{A} / \mathrm{B}$ ratio was standardized dividing the total duratin of accleration by the total duration of movement bursts (Figure 8). ACGs of 15 common fetal disorders were quantitatively analysed with $A / B$ ratios, and compared to the Apgar scores and the numeric long term outcome after births [9].

The short term outcome analysed by 1 and 5 min Apgar score closely correlated A/B ratios, i.e. Y ( 1 min Apgar $)=7.68 \mathrm{X}(\mathrm{A} / \mathrm{B}$ ratio)-1.75, $\mathrm{R}^{2}=0.85, \mathrm{p}<0.001, \mathrm{Y}(5 \mathrm{~min}$ Apgar $)=6.44 \mathrm{X}+0.58, \mathrm{R}^{2}=0.68$, $\mathrm{p}<0.001$, and $\mathrm{Y}$ (numeric long term outcome) $=6.42 \mathrm{X}+0.05, \mathrm{R}^{2}=0.71$, $\mathrm{p}<0.001$. Short and long term outcomes were abnormal, when the $A / B$ ratio was less than 1 . The fact was remarkable that a spastic quadriplegia case was found among cases of lower $\mathrm{A} / \mathrm{B}$ ratio than 1 . Therefore, $\mathrm{A} / \mathrm{B}$ ratio of ACG is a useful parameter to expect fetal short and long term outcomes. Regression equations are listed [9].

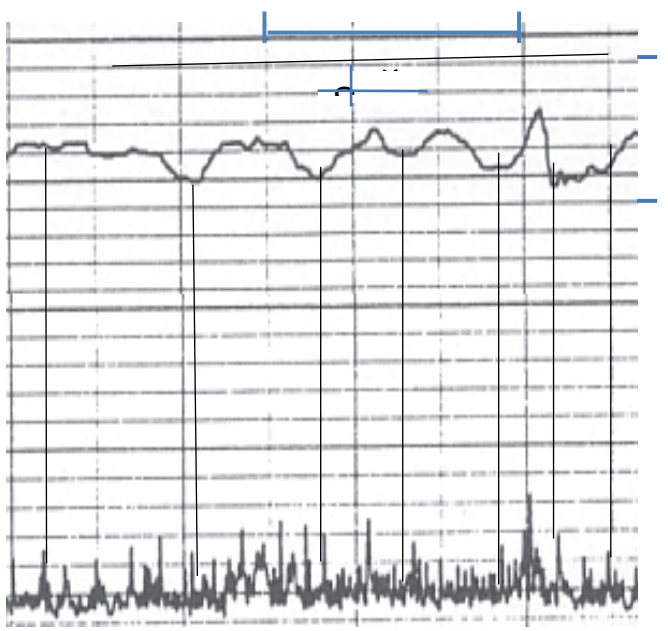

Figure 7: The FHR variability (upper line, LTV) forms variation with the minor fetal movements visualized in an augmented fetal ACG.

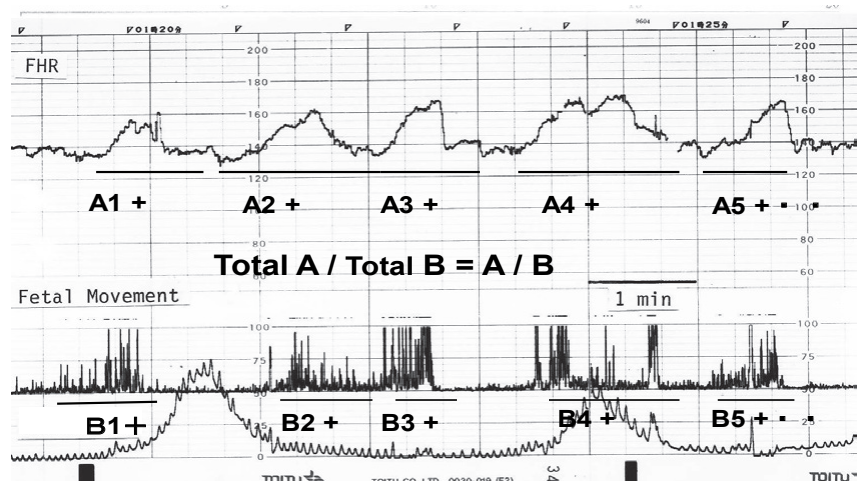

Figure 8: The calculation of $A / B$ ratio in a $A C G$ recorded in active fetal state. 


\section{COMMENT}

\section{Comprehensive evaluaton of the fetus}

Fetal diagnosis with the pattern classification tends to the single item diagnosis but not to cover the total condition of the fetus. In quantitative diagosis, the FHR score predicts Apgar score and umbilical cord arterial blood $\mathrm{pH}$ even in the first stage of labor. Neural netork analyais reports the pathological outcome probability. In the ACG, the $\mathrm{A} / \mathrm{B}$ ratio predicts pathological Apgar score and long term outcome after the birth. Automated comprehensive diagnosis and outcome prediction are big advantage of quantitative analysis of FHR and ACG.

\section{Advantage of fetal movement record}

Since the change of movement signal of ACG is the same as those of fetal movement (Figure 2), the inflence of fetal movement on FHR is precisely evluated, i.e. fetal movement precedes FHR change, e.g. periodic fetal movements provoked sine wave-like FHR changes (Figure 5), where the physiologic sinusoidal FHR is differentiated from the truly ominous sinusoidal heart rate by the ACG. Resting state of the fetus is differentiated by the absence of fetal movement bursts.from non-reactive FHR. The loss of acceleration against feal movement burst shows hypoxic suppression of fetal brain predicting the severe hypoxia.

\section{Frequency analysis of FHR traces}

The tecnique was introduced with the purose to automatically diagnose truly ominous sinusoidal FHR, which appeared without fetal movement record, and quantitatively confirmed by the FFT frequency analysis [11]. It was a surprize to diagnose severe loss of FHR variabilty less than the resting fetal state also by a FFT frequency analysis of FHR record [12].

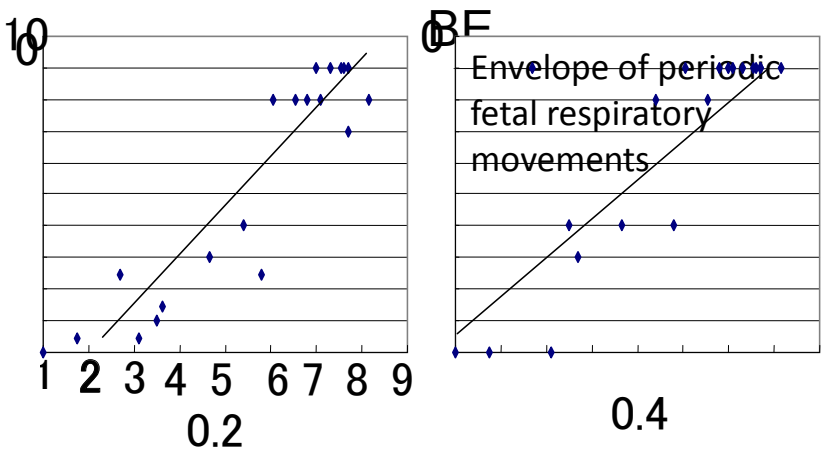

Figure 9: One and $5 \mathrm{~min}$ Apgr scores closely correlated the A/B ratio of ACG recorded during pregnancy in cases of fetal.

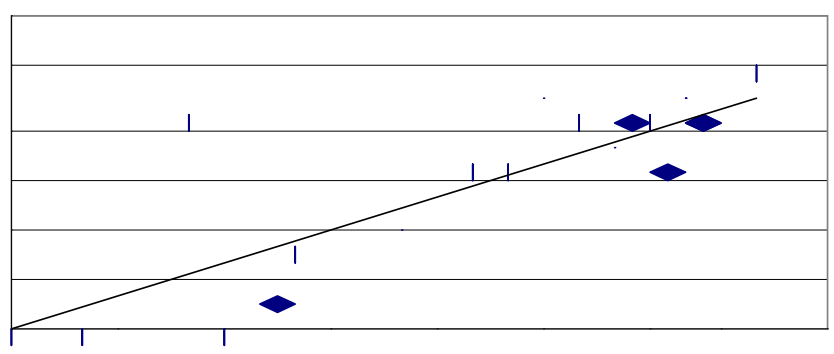

Figure 10: The long term numerical outcome (right) determined using voluntarily defined numerical outcome (left) closely correlated the A/B ratio of ACG recorded in pregnancy [9]. Regression equation is in the text.

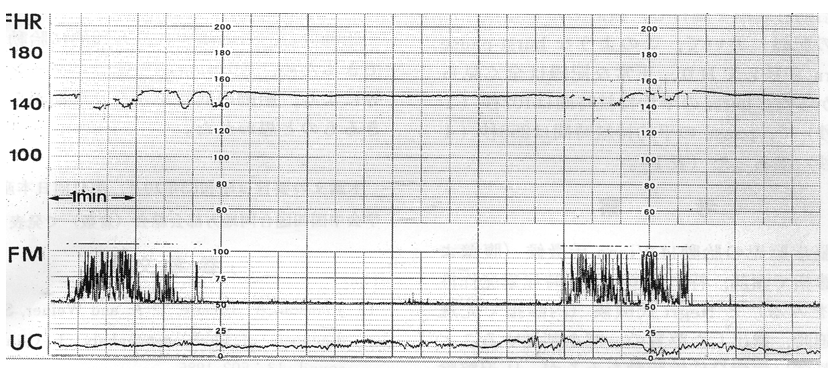

Figure 11: The fetal ACG of an anencepalic fetus. The variability (LTV) is less than $1 \mathrm{bpm}$. There was no acceleration against the frequent fetal movement.
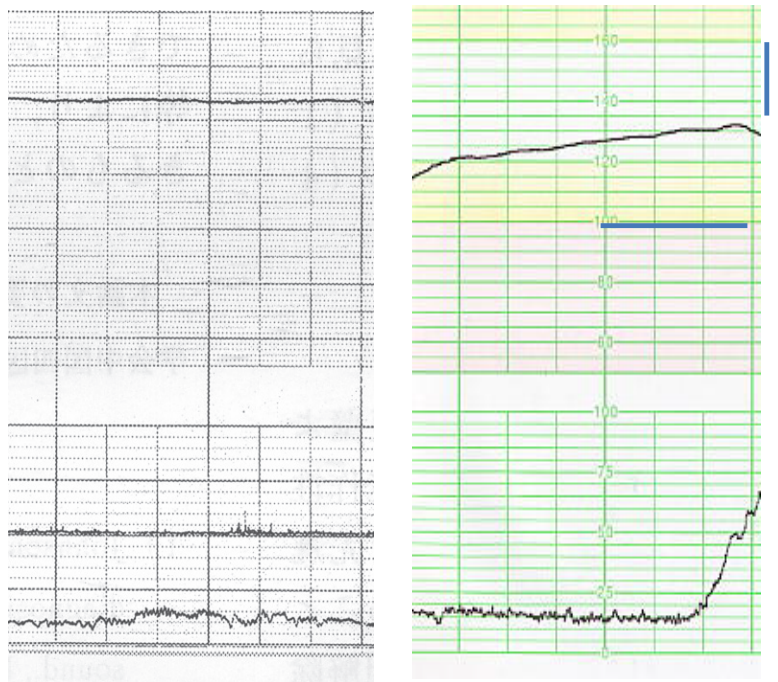

Figure 12: The comparison of CTG ( right) of a severe fetal asphyxia (hypoxia), of which FHR was a late deceleration and the loss of variability (LTV), which was the same as the baseline of the anencepaly (left), of wich LTV ampltude was less than $1 \mathrm{bpm}$. Fetal brain damage in the asphyxia was as severe as the loss of brain in the anencephly [15].

\section{The fetal state in severe loss of variability (LTV)}

Since anencephalic fetal ACG recorded neither acceleration nor LTV, normal fetal brain is responsible to the development of acceleration and LTV (Figures 9-11), but not in the brain cortex because no heart rate change was recognized by the person who developed the triangular acceleration in adult exercise [15], and the fetal acceleration was reported to be produced in the midbrain in the studies on anencephalic fetus [16]. The FHR acceleration disappeared in the non-reactive FHR, while the LTV was preserved, and severe hypoxic FHR changes appear some days later in non-reactive FHR cases, including bradycardia, late deceleration and the loss of LTV. The outcome of C-section in this state was ominous, if compared to reactive FHR [14], i.e. fetal damage was heavy in cases of the loss of LTV, which will be severe brain damage. In an intrapartum fetus of heavy loss of LTV less than $1 \mathrm{bpm}$ similar to anencephalic fetus (Figure 12) whose mother refused C-section and a severely depressed neonate with apnea was vaginally delivered, where 1 min Apgar was 3 due to the brain damage. Therefore, a severe loss of LTV means such brain damage as the loss of bran in anencephalic fetus. A severe hypoxic loss of FHR variability was rare, but the outcome will be ominous with probable $\mathrm{CP}$ caused by neuronal cell necrosis. Thus, the C-section after the severe loss of variability may not guarantee totally healthy neonate, but there will be the risk of possible brain 


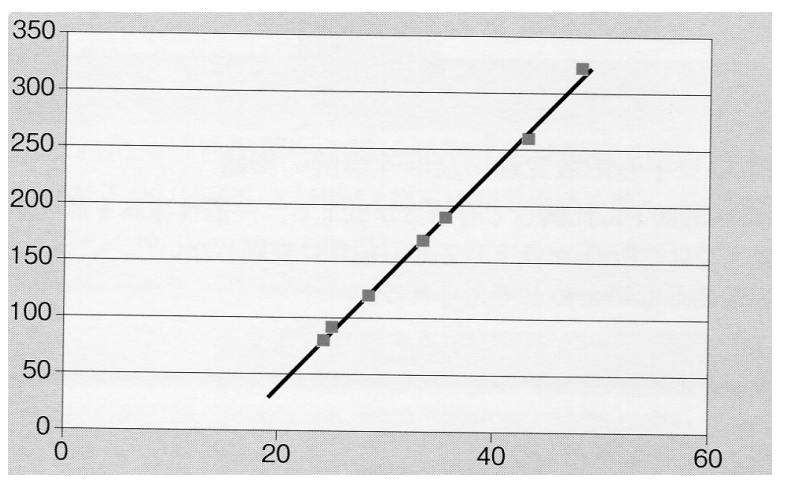

Figure 13: Close relation of heart rate and $\mathrm{PaO}_{2}$ in hypoxic female rabbit.

damage followed by cerebral palsy. That is why earlier C-section is recommended before severe loss of variability [15]. The ideal timing of C-section will be shown after the estimation of hypoxic impact on fetal brain, e.g. by the hypoxic index, which is determied with the hypoxia duration (min) divided by the $\mathrm{PaO}_{2}(\%)$ where nadir FHR (bpm) is adopted instead of $\mathrm{PaO}_{2}$, because of close relation $\left(\mathrm{R}^{2}=1\right)$ in rabbit heart rate and $\mathrm{PaO}_{2}$ (Figure 13) [15]. Since the hypoxic index of 3 fetuses after the loss of variability were $25-26$, a C-section is recommended when the hypoxic index reached 20-24, while this threshold hypoxic index value should be studied in more cases.

\section{Abnormal FHR in general insults without hypoxia}

Abnormal FHR changes were reported in cytomgalovirus (CMV) infected fetus [17] and in congenital syphilis [18]. Therapeutic decision still seemes controversial in the occasion because of no hypoxia. However, as discussed above, such abnormal FHR as severe loss of LTV is caused by fetal brain damage, the FHR changes can develop by viral or bacterial toxin or oher general insults. As the loss of LTV means severe damage of fetal brain, FHR changes in various general insults should be treated by the same strategy as hypoxic FHR changes, i.e. $\mathrm{C}$-section before the loss of variability. The A/B ratio of ACG will be helpful for the outcome prediction.

\section{Computerization of fetal monitoring}

The FHR score calculation, neural network analysis of FHR, frequency analysis of FHR, discusssed in this report, were progrmmed and working in the centralized fetal monitoring, improving perinatal status $[5,6,10]$. Quantified ACG and hypoxic index will be added to the system in the near future. An attending obstetrician will input continuous FHR into the computer system, and receive analyzed results rapidly and directly from the system. The time consuming visual CTG analysis will be changed to the computerization to receive objective, comprehensive and precise diagnostic data [6].

\section{Conclusion}

Since the ACG and its quantitative analysis proposed new field of fetal evaluation, which is totally objective in quantitative as well as visual analysis of ACG as discussed in this report, the subjective visual FHR pattern diagnosis, whch was vague with big interobserver difference, is definitely improved and the fetal management is greatly progressed by the introduction of quantified FHR analysis and fetal ACG, where C-section was recommeded to be performed before the loss of FHR variability. In addition, computerized automatic feal diagnosis, which utilizes various quantified analyses, extensively improves obstetric statuses.

\section{References}

1. Maeda K (1984) Studies on new ultrasonic Doppler fetal actograph, and continuous recording of fetal movement. Acta Obstet Gynecol Jpn 36: 280-288.

2. Maeda K (2004) Fetal monitoring and actocardiogram in the evaluation of fetal behavior. Ultrasound Rev Obstet Gynecol 4: 12-25.

3. Maeda K , Kimura S, Nakano H (1969) Pathophysiology of Fetus, 1969, Fukuoka Printing, Fukuoka, Proc XXI Annual Conv Jap Obstet and Gynecol Society, Kanazawa, 1969.

4. Hon EH (1968) An Atlas of Fetal Heart Rat Patterns. Harty Press, New Haven, Connecticat 305

5. Maeda K, Noguchi Y, Matsumoto F, Nagasawa T (2006) Quantitative fetal heart rate evaluation without pattern classification: FHR score and artificial neural network analysis. Network 21: 127-141.

6. Kazuo Maeda, Masaji Utsu, Yasuaki Noguchi, Fujihiko Matsumoto, Takash Nagasawa T, et al. (2012) Central computerized automatic fetal heart rate diagnosis with a rapid and direct alarm system.The Open Medical Devices Journal 4: 28-33.

7. Nijhuis JG (1998) Fetal behavioral states, in FA Chervenak, GC Isacson, S Campbell, eds. Ultrasound in Obstetrics and Gynecology, Vol 1, Boston, Little \& Brown, 1998: 447-455

8. Ito T, Maeda K, Takahashi H, Nagata N, Nakajima K, et al. (1994) Differentiation between physiologic and pathologic sinusoidal FHR pattern by fetal actocardiogram. J Perinat Med 22:39-43.

9. Maeda K, Iwabe T, Yoshida S, Ito T, Minagawa Y, et al. (2009) Detailed multigrade evaluation of fetal disorders with the quantified actocardiogram. J Perinat Med 37: 392-396.

10. Maeda K, Utsu M, Makio A, Serizawa M, Noguchi Y, et al. (1998) Neural network computer analysis of fetal heart rate. J Maternal Fetal Invest 8: 163-171.

11. Maeda K, Nagasawa T (2005) Automatic computerized diagnosis of fetal sinusoidal heart rate. Fetal Diagn Ther 20: 328-334.

12. Maeda K, Nagasawa T (2010) Loss of FHR variability diagnosed by frequency analysis. J Perinat Med 38: 197-201.

13. Maeda K (2005) Quantitative studies on fetal actocardiogram. Croat Med J 46 792-796.

14. Teshima N (1993) Non-reactive pattern diagnosed by ultrasonic Doppler fetal actocardiogram and outcome of the fetuses with non-reactive pattern. Nihon Sanka Fujinka Gakkai Zasshi 45: 423-430.

15. Maeda K (2012) Actocardiographic analysis of fetal hypoxia detected by the bradycardia, loss of fetal heart rate acceleration and long term variability. J Health Med Inform.

16. Terao T, Kawashima Y, Noto H, Inamoto Y, Lin TY, et al. (1984) Neurological control of fetal heart rate in 20 cases of anencephalic fetuses. Am J Obstet Gynecol 140: 201-208.

17. Kaneko M, Sameshima H, Ikeda T, Ikenoue T, Minematsu T (2004) Intrapartumfetal heart rate monitoring in cases of cytomegalovirus infection. Am J Obstet Gynecol 191: 1257-1262.

18. Kakogawa J, Sadalsuki M, Masuya N, Gomibuchi H, Minoura S, et al. (2011) Prolonged fetal bradycardia as the presenting sign in congenital syphilis complicated by necrotizing funisitis: a case report. ISRN Obstet Gynecol 3. 